\title{
Universal Exchange Algebra for Bloch Waves and Liouville Theory
}

\section{O. Babelon}

Laboratoire de Physique Théorique et Hautes Energies-Paris, Université Pierre et Marie Curie, Tour $161^{\text {er }}$ étage, 4 Place Jussieu, F-75252 Paris Cedex 05, France

Received February 11, 1991

\begin{abstract}
We derive a universal formula for the exchange algebra in the Bloch wave basis. The main tool we use is a lattice version of the Coulomb gas picture of conformal field theory, making its quantum group structure explicit from the very beginning. Calulations are then reduced to a factorization problem in $\mathscr{U}_{q}\left(s l_{2}\right)$.
\end{abstract}

\section{Introduction}

There is an intimate connection between Liouville theory, conformal field theory and quantum groups. As a unifying feature, we will take the Schroedinger equation

$$
\left(\partial_{z}^{2}-\mathscr{U}\right) \xi=0 .
$$

The relation to Liouville's equation already appeared in Poincaré [1] and is as follows. Let $\xi^{1}$ and $\xi^{2}$ be two linearly independent solutions of Eq. (1) with their Wronskian normalized to one. Setting

$$
e^{-\frac{1}{2} \varphi}=\xi^{1} \bar{\xi}^{2}-\xi^{2} \bar{\xi}^{1},
$$

it is straightforward to check that the field $\varphi$ satisfies Liouville's equation

$$
\partial_{z} \partial_{\bar{z}} \varphi=2 e^{\varphi} .
$$

The meaning of Eq. (1) in conformal field theory may be easily understood by looking at the transformation properties of this equation under conformal transformations. Let us change coordinates $z \rightarrow z(w)$. If $\xi$ behaves like a differential of weight $-\frac{1}{2}$ and $\mathscr{U}$ as a Schwarzian connection, i.e.

$$
\begin{aligned}
\xi(w) d w^{-\frac{1}{2}} & =\xi(z) d z^{-\frac{1}{2}}, \\
\mathscr{U}(w) d w^{2} & =\mathscr{U}(z) d z^{2}+\frac{1}{2}\{z, w\} d z^{2},
\end{aligned}
$$


where $\{z, w\}$ denotes the Schwarzian derivative

$$
\{z, w\}=\frac{z^{\prime \prime \prime}}{z^{\prime}}-\frac{3}{2}\left(\frac{z^{\prime \prime}}{z^{\prime}}\right)^{2},
$$

then the quantity $\left(\partial_{z}^{2}-\mathscr{U}\right) \xi$ behaves like a differential of weight $\frac{3}{2}$ [2]

$$
d w^{\frac{3}{2}}\left(\partial_{w}^{2}-\mathscr{U}(w)\right) \xi(w)=d z^{\frac{3}{2}}\left(\partial_{z}^{2}-\mathscr{U}(z)\right) \xi(z) .
$$

This covariance property is strongly reminiscent of null vectors. More precisely, let $V_{n}$ be generators satisfying the Virasoro algebra

$$
\left[V_{n}, V_{m}\right]=(n-m) V_{n+m}+\frac{1}{12} c\left(n^{3}-n\right) \delta_{n,-m} .
$$

Consider a representation of $V_{n}$ acting on some Hilbert space $\mathscr{H}$. Let

$$
T(z)=\sum_{n \in Z} z^{-n-2} V_{n}
$$

As it is well known, on the set of operators $\Theta(z)$ acting on $\mathscr{H}$ we may define a representation $V_{n}(z)$ of the Virasoro algebra with the same central charge as in Eq. (2) by writing the short distance expansion [3]

$$
T(w) \Theta(z)=\sum_{n \in Z} \frac{V_{n}(z) \Theta(z)}{(w-z)^{n+2}} .
$$

In this representation, the highest weight vectors are the conformal operators. In fact

$$
\left[V_{n}, \xi(z)\right]=z^{n}\left(z \frac{d}{d z}+(n+1) \Delta\right) \xi(z)
$$

translates into the highest weight condition on $\xi(z)$,

$$
\begin{aligned}
& V_{n}(z) \xi(z)=0, \quad n>0, \\
& V_{0}(z) \xi(z)=\Delta \xi(z) .
\end{aligned}
$$

If $\Delta$ takes one of the values given by Kac's determinant formula, the Verma module built on $\xi(z)$ is reducible and there exists null vectors. Let us parametrize the central charge in Eq. (2) as

$$
c=1+6\left(\frac{\pi}{\gamma}+\frac{\gamma}{\pi}+2\right)
$$

so that $c>25$ corresponds to $\gamma$ real $>0$, while $c<1$ corresponds to $\gamma$ real $<0$. Kac's dimensions are given by [4]

$$
\Delta_{j, j^{\prime}}=-\left[j \sqrt{\frac{\gamma}{\pi}}+j^{\prime} \sqrt{\frac{\pi}{\gamma}}\right]\left[(j+1) \sqrt{\frac{\gamma}{\pi}}+\left(j^{\prime}+1\right) \sqrt{\frac{\pi}{\gamma}}\right],
$$

where $j, j^{\prime}$ are integers of half integers. For $j=\frac{1}{2}, j^{\prime}=0$, the decoupling condition of the level two null vector reads

$$
\left[V_{-1}^{2}(z)+\frac{\gamma}{\pi} V_{-2}(z)\right] \xi(z)=0
$$


Working on the cylinder instead of the sphere, i.e. setting $z=e^{i x}, \xi(x)=z^{\Delta} \xi(z)$, we get $[5,3]$

$$
\partial_{x}^{2} \xi(x)-: \mathscr{U}(x) \xi(x):=0
$$

where

$$
\mathscr{U}(x)=\frac{\gamma}{\pi} z^{2} T(z)-\frac{1}{4}-\frac{1}{2} \frac{\gamma}{\pi}-\frac{3}{16}\left(\frac{\gamma}{\pi}\right)^{2} .
$$

The double dots mean writing the operator $V_{n}$ on the left if $n<0$, on the right if $n>0$ and sharing $V_{0}$ equally on both sides.

The semi-classical limit corresponds to $\gamma \rightarrow 0$. In this limit

$$
\Delta_{j, 0} \rightarrow-j \text {. }
$$

For $j=1 / 2$, the corresponding null vector also has a finite limit: we simply get back Eq. (1). So, the Schrödinger equation is the semi-classical limit of the decoupling condition for the null vector of weight $\Delta_{1 / 2,0}$.

More generally, for arbitrary $j$ the analog of Eq. (1) is a differential equation of order $2 j+1$,

$$
\left(\partial^{2 j+1}+\sum_{i=0}^{2 j-1} U_{i} \partial^{i}\right) \xi^{(j)}=0
$$

where the coefficients $\mathscr{U}_{i}$ are local functions of the energy momentum tensor $\mathscr{U}$ and its derivatives [21].

Finally, one has to explain the relation with quantum groups. Equation (4) has a basis of $2 j+1$ linearly independent solutions $\xi_{m}^{(j)}(x)$. These solutions are functionals of $\mathscr{U}$. The semi-classical limit of Eq. (2) defines on $\mathscr{U}$ a Poisson bracket

$$
\{\mathscr{U}(x), \mathscr{U}(y)\}=2 \gamma\left(2 \mathscr{U}(x) \partial_{x}+\mathscr{U}^{\prime}(x)-\frac{1}{2} \partial_{x}^{3}\right) \delta(x-y),
$$

and we may compute the Poisson brackets of the $\xi_{m}^{(j)}$. (Poisson brackets are calculated at time $t=0$. We assume $x \in S^{1}$.) For a suitable choice of basis this Poisson bracket takes the simple form

$$
\left\{\xi^{(j)}(x) \stackrel{\otimes}{,} \xi^{\left(j^{\prime}\right)}(y)\right\}=-\frac{\gamma}{2} \xi^{(j)}(x) \otimes \xi^{\left(j^{\prime}\right)}(y)\left[\theta(x-y) r^{+}+\theta(y-x) r^{-}\right],
$$

where the tensorial notation means that $\xi^{(j)}$ is a line vector of components $\xi_{m}^{(j)}$ and by definition $\left\{\xi^{(j)}(x)^{\otimes}, \xi^{\left(j^{\prime}\right)}(y)\right\}_{m m^{\prime}}=\left\{\xi_{m}^{(j)}(x), \xi_{m^{\prime}}^{\left(j^{\prime}\right)}(y)\right\}$. The matrices $r^{ \pm}$are solutions of the classical Yang-Baxter equation and are given by

$$
\begin{aligned}
& r^{+}=H \otimes H+4 E_{+} \otimes E_{-}, \\
& r^{-}=-H \otimes H-4 E_{-} \otimes E_{+} .
\end{aligned}
$$

They are expressed in terms of the generators $H, E_{ \pm}$of $s l_{2}$

$$
\begin{aligned}
{\left[H, E_{ \pm}\right] } & = \pm 2 E_{ \pm}, \\
{\left[E_{+}, E_{-}\right] } & =H .
\end{aligned}
$$

In Eq. (5) one has to use the adequate representations of $s l_{2}$ in the two factors of the tensor product. A simple way to prove this formula is to use the relation with Liouville equation and exploit its integrable structure [6]. One 
should stress that in another basis the result Eq. (5) may look quite different. Moreover the basis where it assumes the simple form Eq. (5) is by no means unique.

Since the coefficients in Eq. (4) are periodic functions of $x$ there is one basis which is quite natural and plays an important role. It is the basis of quasiperiodic solutions (Bloch waves),

$$
\psi^{(j)}(x+2 \pi)=\psi^{(j)}(x) e^{2 \pi P_{0} H},
$$

where $P_{0}$ is the Bloch momentum and $H$ is a traceless diagonal matrix. The Poisson bracket of the $\psi$ 's can be computed and we find

$$
\begin{aligned}
\left\{\psi^{(j)}(x) \stackrel{\otimes}{,} \psi^{\left(j^{\prime}\right)}(y)\right\}= & -\frac{\gamma}{4} \psi^{(j)}(x) \otimes \psi^{\left(j^{\prime}\right)}(y) \\
& \cdot\left[\left(r^{+}-r^{-}\right) \varepsilon(x-y)-\frac{(A+D)}{(A-D)}\left(r^{+}+r^{-}\right)\right],
\end{aligned}
$$

where

$$
A=e^{2 \pi P_{0}}, \quad D=e^{-2 \pi P_{0}} .
$$

Equations $(5,8)$ are universal formulae in the sense that Eqs. $(6,7)$ give an expression of $r^{ \pm}$in terms of the generators of $s l_{2}$ only, independent of the representations.

One purpose of this work is to derive the quantum version of Eqs. $(5,8)$. It is not difficult to write the quantum generalization of Eq. (5),

$$
\xi_{1}^{(j)}(x) \xi_{2}^{\left(j^{\prime}\right)}(y)=\xi_{2}^{\left(j^{\prime}\right)}(y) \xi_{1}^{(j)}(x) R_{12}^{ \pm}(q)
$$

where again we have used the now standard tensor notation

$$
\left[\xi_{1}^{(j)}(x) \xi_{2}^{\left(j^{\prime}\right)}(y)\right]_{m m^{\prime}}=\xi_{m}^{(j)}(x) \xi_{m^{\prime}}^{\left(j^{\prime}\right)}(y), \quad\left[\xi_{2}^{\left(j^{\prime}\right)}(y) \xi_{1}^{(j)}(x)\right]_{m m^{\prime}}=\xi_{m^{\prime}}^{\left(j^{\prime}\right)}(y) \xi_{m}^{(j)}(x) .
$$

The sign $\pm=\varepsilon(x-y)$ so that by consistency we must have $R_{12}^{-}(q)=$ $\left[R_{21}^{+}(q)\right]^{-1}$. Now $R_{12}^{ \pm}(q)$ are solutions of the quantum Yang-Baxter equation. Their universal form is [7],

$$
\begin{aligned}
& R_{12}^{+}(q)=q^{\frac{1}{2} H \otimes H} \sum_{i=0}^{\infty}\left(q-q^{-1}\right)^{i} \frac{q^{-\frac{i(i+1)}{2}}}{[i] !} q^{\frac{i}{2} H} E_{+}^{i} \otimes q^{-\frac{i}{2} H} E_{-}^{i}, \\
& R_{12}^{-}(q)=q^{-\frac{1}{2} H \otimes H} \sum_{i=0}^{\infty}(-1)^{i}\left(q-q^{-1}\right)^{i} \frac{q^{\frac{i(i+1)}{2}}}{[i] !} q^{\frac{i}{2} H} E_{-}^{i} \otimes q^{-\frac{i}{2} H} E_{+}^{i},
\end{aligned}
$$

where now $H, E_{ \pm}$denote the generators of the quantum group $\mathscr{U}_{q}\left(s l_{2}\right)$,

$$
\begin{gathered}
{\left[H, E_{ \pm}\right]= \pm 2 E_{ \pm},} \\
{\left[E_{+}, E_{-}\right]=\frac{q^{H}-q^{-H}}{q-q^{-1}} .}
\end{gathered}
$$

The value of the parameter $q$ which fits with the semi-classical limit is $q=e^{-i \gamma}$. It is associated to the fields of dimensions $\Delta_{j, 0}$. Due to the symmetry $\gamma \rightarrow \pi^{2} / \gamma$ in Eq. (3) one could take also $q=e^{-i \frac{\pi^{2}}{\gamma}}$, and all we say would apply to the fields with dimensions $\Delta_{0, j}$. 
It is not so easy to guess what is the quantum generalization of Eq. (8). The answer to this question is the main result of this paper. It is given by the following

\section{Theorem 1.}

$$
\psi_{1}^{(j)}(x) \psi_{2}^{\left(j^{\prime}\right)}(y)=\psi_{2}^{\left(j^{\prime}\right)}(y) \psi_{1}^{(j)}(x) \mathscr{R}_{12}^{ \pm}\left(q, P_{0}\right)
$$

where

$$
\mathscr{R}_{12}^{ \pm}\left(q, P_{0}\right)=F_{21}^{-1}\left(q, P_{0}\right) R_{12}^{ \pm}(q) F_{12}\left(q, P_{0}\right)
$$

and

$$
\begin{aligned}
F_{12}\left(q, P_{0}\right)= & \sum_{k=0}^{\infty}\left(q-q^{-1}\right)^{k} \frac{(-1)^{k}}{[k] !} \\
& \cdot \frac{A^{k}}{\prod_{v=k}^{2 k-1}\left(A q^{H_{2}+v}-D q^{-H_{2}-v}\right)} q^{\frac{k}{2}\left(H_{1}+H_{2}\right)} E_{+}^{k} \otimes E_{-}^{k} .
\end{aligned}
$$

In these formulae, $P_{0}$ is the quantum Bloch momentum

$$
\psi^{(j)}(x+2 \pi)=\psi^{(j)}(x) e^{2 \pi P_{0} H} q^{\frac{1}{2} H^{2}} .
$$

As in all this paper $A=e^{2 \pi P_{0}}$ and $D=e^{-2 \pi P_{0}}$.

For $j=j^{\prime}=1 / 2$ this exchange algebra was first obtained in [5].

Once the $\psi$-basis is constructed, one can try to define the $\xi$-basis out of it. One can do it using a change of basis depending only on $P_{0}$

Theorem 2. Let

$$
\xi^{(j)}(x)=\psi^{(j)}(x) M\left(P_{0}\right)
$$

where

$$
M\left(P_{0}\right)=\sum_{n, m=0}^{\infty} \frac{(-1)^{m} A^{m} q^{\frac{1}{2} n(n-1)+m(n-m)}}{[n] ![m] ! \prod_{\nu=1}^{n}\left(A q^{\nu}-D q^{-v}\right)} E_{+}^{n} E_{-}^{m} q^{\frac{1}{2(n+m) H}},
$$

then $\xi^{(j)}(x)$ satisfies $E q$. (9).

The $\xi$-basis and the $\psi$-basis both have their own merits. The $\xi$-basis is best suited to discuss the quantum group structure of the theory, while the $\psi$-basis is important for the following reason. One can show that the Bloch momentum $P_{0}$ commutes with the Virasoro generators $V_{n}$. Therefore one can split the representation space $\mathscr{H}$ into a sum of subspaces $\mathscr{H}_{\omega_{j}}$ indexed by the eigenvalues $\omega_{j}$ of $P_{0}$

$$
H=\bigoplus_{j} \mathscr{H}_{\omega_{j}}
$$

Each $\mathscr{H}_{\omega_{j}}$ carries a representation of the Virasoro algebra. The distinguished property of the Bloch waves is that they simply intertwine between these spaces according to the relation

$$
P_{0} \psi^{(j)}(x)=\psi^{(j)}(x)\left[P_{0}-\frac{i \gamma}{2 \pi} H\right] .
$$


Let us now a briefly outline the content of this paper. One noticeable aspect of our work is the use of a lattice regulator [8]. This yields a new nonultralocal structure described in Sects. 2 and 3, which is at the basis of our construction. Of course the introduction of a lattice cutoff breaks conformal invariance, but the cutoff is introduced precisely in such a way that the exchange algebra, which is our main concern here, remains unchanged. The advantage of this method is that the quantum group structure is made explicit from the very beginning. With this tool at hand, we give in Sect. 4 a precise definition of the Bloch waves at the quantum level and we calculate their commutation relations in Sect. 5. The $\xi$-basis is constructed in Sect. 6.

All we do with one chirality can also be done with the other chirality. In Sect. 7 we construct the Bloch waves $\bar{\psi}^{(j)}(x)$ for the second chirality and we give their exchange algebra. Finally, once the two chiralities are defined one can combine them to reconstruct a set of periodic and local fields. This is done in Sect. 8. In the conclusion, we discuss some implications of our results for the quantum Liouville theory. We also give a free field representation of the basic non-ultralocal algebra on the lattice which we will be useful in forthcoming applications.

\section{Classical Theory}

In this section, we sketch the main steps of the construction yielding to the Poisson bracket Eq. (8) for the quasi-periodic solutions of Eq. (4). We refer to [9] for the detailed proofs. We first replace the single differential equation of order $2 j+1$ by a system of first order equations. A very convenient such system is the Drinfeld-Sokolov system $[10,11]$,

$$
\partial_{x} Q=\left(P(x) H-E_{+}\right) Q .
$$

Here $P(x)$ is a periodic field. Poisson bracket is defined by

$$
\{P(x), P(y)\}=\gamma \delta^{\prime}(x-y) .
$$

Taking $H$ and $E_{+}$in a spin $j$ representation of $s l_{2}$, we find that the matrix elements of the first line of $Q(x)$

$$
\sigma^{(j)}(x)=\left\langle\lambda_{\max }^{(j)}\right| Q(x)
$$

satisfy a differential of order $2 j+1$ which is precisely our Eq. (4). For instance in the spin $1 / 2$ representation

$$
H=\left(\begin{array}{rr}
1 & 0 \\
0 & -1
\end{array}\right), \quad E_{+}=\left(\begin{array}{ll}
0 & 1 \\
0 & 0
\end{array}\right), \quad E_{-}=\left(\begin{array}{ll}
0 & 0 \\
1 & 0
\end{array}\right)
$$

we get

$$
\left(\partial_{x}^{2}-\mathscr{U}\right) \sigma^{(1 / 2)}(x)=0
$$

with

$$
\mathscr{U}(x)=P^{2}(x)+P^{\prime}(x) .
$$


Consider the solution of Eq. (10) normalized by the condition $Q(0)=1$. The monodromy matrix $S$ is defined by

$$
Q(x+2 \pi)=Q(x) S, \quad S=Q(2 \pi) .
$$

To construct the Bloch waves we diagonalize $S$. Let

$$
S=g^{-1} e^{2 \pi P_{0} H} g,
$$

the matrix $g$ is uniquely determined if we require it to be strictly upper triangular. Then we set

$$
\psi^{(j)}(x)=\left\langle\lambda_{\max }^{(j)}\right| Q(x) g^{-1} \varrho,
$$

where $\varrho$ is a diagonal matrix. These $\psi^{(j)}(x)$ have diagonal monodromy

$$
\psi^{(j)}(x+2 \pi)=\psi^{(j)}(x) e^{2 \pi P_{0} H} .
$$

The diagonal matrix $\varrho$ is chosen as follows

$$
\varrho=e^{-K+H} e^{\theta H} .
$$

The constant $K_{+}$is defined by

$$
\int_{0}^{x} P(y) d y=K_{+}+P_{0} x+\sum_{n \neq 0} \frac{P_{n}}{i n} e^{i n x},
$$

where we have introduced the Fourier decomposition of $P(x)$

$$
P(x)=\sum_{n} P_{n} e^{i n x} .
$$

The role of $K_{+}$is to eliminate from $\psi^{(j)}(x)$ all the remaining dependence in the normalization point $x=0$. The variable $\theta$ is the conjugate variable of $P_{0}$,

$$
\left\{\theta, P_{0}\right\}=\frac{\gamma}{2 \pi} .
$$

With these choices, we have shown in [9] that $\psi^{(j)}(x)$ is a conformal object of weight $-j$ and the exchange algebra is the one described in Eq. (8). Here we simply recall the following relations which will be used later. For $0<x$, $y<2 \pi$ we have

$$
\begin{aligned}
\{Q(x) \stackrel{\otimes}{,} Q(y)\}= & \frac{\gamma}{2} Q(x) \otimes Q(y)\left\{\theta ( x - y ) \left[-r+Q^{-1}(y)\right.\right. \\
& \left.\otimes Q^{-1}(y)(r-H \otimes H) Q(y) \otimes Q(y)\right] \\
& +\theta(y-x)\left[-r+Q^{-1}(x) \otimes Q^{-1}(x)(r+H \otimes H)\right. \\
& \cdot Q(x) \otimes Q(x)]\},
\end{aligned}
$$

where $r$ is either $r^{+}$or $r^{-}$. We will need also the Poisson brackets of the matrix $\varrho$,

$$
\begin{aligned}
& \{\varrho \stackrel{\otimes}{,} \varrho\}=0, \\
& \{Q(x) \stackrel{\otimes}{,} \varrho\}=-\frac{\gamma}{2} Q(x) \otimes \varrho(H \otimes H), \quad 0<x<2 \pi, \\
& \{S \stackrel{\otimes}{\varrho} \varrho\}=-\frac{\gamma}{2}[S \otimes \varrho H \otimes H+(H \otimes H) S \otimes \varrho] .
\end{aligned}
$$




\section{Quantum Theory on the Lattice}

To generalize our analysis to the quantum case one has to define a suitable ordering for operators. A natural choice would be normal ordering. It has the advantage of preserving conformal invariance [5].

Another possibility, the one we will follow, is to go to the lattice and use the lattice ordering [8]. Conformal invariance is lost (in fact it is only deformed $[15,18])$, but this does not matter as far as the exchange algebra is concerned. It has the advantage that the quantum group structure is made explicit from the very beginning.

To go to the lattice, the first step is to discretize Eq. (10). As usual, we set

$$
Q_{n}=L_{n} Q_{n-1} .
$$

The problem is to define a suitable Poisson bracket for the $L_{n}$ 's. We do it by requiring that $Q_{n}$ satisfies the lattice version of Eq. (14),

$$
\begin{aligned}
\left\{Q_{n} \stackrel{\otimes}{,} Q_{m}\right\}= & \frac{\gamma}{2} Q_{n} \otimes Q_{m} \\
& \cdot\left\{\theta(n-m)\left[-r+Q_{m}^{-1} \otimes Q_{m}^{-1}(r-H \otimes H) Q_{m} \otimes Q_{m}\right]\right. \\
& \left.+\theta(m-n)\left[-r+Q_{n}^{-1} \otimes Q_{n}^{-1}(r+H \otimes H) Q_{n} \otimes Q_{n}\right]\right\} .
\end{aligned}
$$

This condition ensures that the exchange algebra on the lattice will be the same as in the continuum. Writing $L_{n}=Q_{n} Q_{n-1}^{-1}$, we find the Poisson bracket algebra of the $L_{n}$ 's,

$$
\begin{aligned}
\left\{L_{n} \otimes\right. & \otimes \\
\left.L_{m}\right\}= & \frac{\gamma}{2} \delta_{n m}\left[r, L_{n} \otimes L_{m}\right] \\
& -\frac{\gamma}{2} \delta_{n, m+1} L_{n} \otimes 1(H \otimes H) 1 \otimes L_{m} \\
& +\frac{\gamma}{2} \delta_{n, m-1} 1 \otimes L_{m}(H \otimes H) L_{n} \otimes 1 .
\end{aligned}
$$

Vice versa if $L_{n}$ satisfy Eq. (20) then

$$
Q_{n}=L_{n} L_{n-1} \ldots L_{1}, \quad n<N,
$$

satisfy Eq. (19). The relations Eq. (20) constitute a non-ultralocal generalization of the usual ultralocal formulae which are at the basis of the Hamiltonian approach of the Inverse Scattering Method [12].

Next we consider a lattice with $N$ sites and periodic boundary conditions. We define the monodromy matrix $S$

$$
S=L_{N} L_{N-1} \ldots L_{1}
$$

so that

$$
Q_{N+n}=Q_{n} S,
$$

remembering that $\left\{L_{1} \stackrel{\otimes}{,} L_{N}\right\} \neq 0$ (in contrast to the ultralocal case), one can show that

$$
\begin{aligned}
& \left\{Q_{n} \stackrel{\otimes}{S} S\right\}=\frac{\gamma}{2} Q_{n} \otimes S\left[-r+Q_{n}^{-1} \otimes Q_{n}^{-1}(r+H \otimes H) Q_{n} \otimes Q_{n}\right. \\
& \left.-H \otimes S^{-1} H S\right] \text {, } \\
& \left\{S^{\otimes}, S\right\}=\frac{\gamma}{2} S \otimes S\left[-r+S^{-1} \otimes S^{-1} r S \otimes S\right. \\
& \left.+S^{-1} H S \otimes H-H \otimes S^{-1} H S\right] .
\end{aligned}
$$


If we add to Eqs. $(19,21,22)$ the obvious lattice analog of Eqs. (15-17), one can repeat on the lattice the same analysis as the one performed in [9] and we get the lattice version of Eq. (8),

$$
\begin{aligned}
\left\{\psi_{n}^{(j)} \stackrel{\otimes}{,} \psi_{m}^{\left(j^{\prime}\right)}\right\}= & -\frac{\gamma}{4} \psi_{n}^{(j)} \otimes \psi_{m}^{\left(j^{\prime}\right)} \\
& \cdot\left[\left(r^{+}-r^{-}\right) \varepsilon(n-m)-\frac{(A+D)}{(A-D)}\left(r^{+}+r^{-}\right)\right] .
\end{aligned}
$$

So the exchange algebra is the same on the lattice and in the continuum, as expected.

To quantize the theory, all we have to do is to give the quantum version of Eq. (20). Introducing the notation

$$
L_{1 n}=L_{n} \otimes I, \quad L_{2 n}=I \otimes L_{n},
$$

a natural quantum generalization of Eq. (20) is

$$
\begin{aligned}
R_{12} L_{1 n} L_{2 n} & =L_{2 n} L_{1 n} R_{12}, \\
L_{1 n} L_{2, n+1} & =L_{2, n+1} A_{12} L_{1 n},
\end{aligned}
$$

where

$$
\begin{aligned}
& R_{12}=1-\frac{i \gamma}{2} r+O\left(\gamma^{2}\right), \\
& A_{12}=1+\frac{i \gamma}{2} H \otimes H+O\left(\gamma^{2}\right) .
\end{aligned}
$$

We will define the quantum theory by requiring that $R_{12}$ is a solution of the quantum Yang-Baxter equation

$$
R_{12} R_{13} R_{23}=R_{23} R_{13} R_{12}
$$

and we take

$$
A_{12}=q^{-\frac{1}{2} H \otimes H}, \quad q=e^{-i \gamma} .
$$

Let us call $R_{12}^{ \pm}$the two solutions of the Yang-Baxter equation whose classical limits are $r_{12}^{ \pm}$respectively. Applying the automorphism $\sigma(x \otimes y)=y \otimes x$ to Eqs. $(23,24)$ we also get

$$
\begin{aligned}
{\left[R_{21}\right]^{-1} L_{1 n} L_{2 n} } & =L_{2 n} L_{1 n}\left[R_{21}\right]^{-1}, \\
L_{2 n} L_{1, n+1} & =L_{1, n+1} A_{12} L_{2 n} .
\end{aligned}
$$

Since $R_{12}^{-}=\left[R_{21}^{+}\right]^{-1}$, we see that we may use indifferently $R_{12}^{+}$or $R_{12}^{-}$in Eq. (23).

As in the ultralocal case, the crucial property of Eqs. $(23,24)$ is that they can be integrated. For this however, one has to change slightly the definition of $Q_{n}$. We define

$$
Q_{n}=L_{n} B L_{n-1} B \cdots B L_{1},
$$

where $B$ is the diagonal matrix

$$
B=q^{-\frac{1}{4} H^{2}} .
$$


We also introduce the monodromy matrix $S$

$$
S=L_{N} B L_{N-1} \cdots B L_{1}
$$

so that if the lattice is periodic with $N$ sites we have

$$
Q_{n+N}=Q_{n} B S \text {. }
$$

Proposition 3.1. Let $L_{n}$ satisfying the relations Eqs. $(23,24)$ and $Q_{n}, S$ be defined as in Eqs. $(25,27)$. Then

1) If $n<N$,

$$
R_{12} Q_{1 n} Q_{2 n}=Q_{2 n} Q_{1 n} R_{12}
$$

2) If $n>m,(n, m<N)$,

$$
Q_{1 n} Q_{1 m}^{-1} A_{21} R_{12} Q_{1 m} Q_{2 m}=Q_{2 m} Q_{1 n} R_{12} .
$$

3) If $n=N, m<N$,

$$
S_{1} Q_{1 m}^{-1} A_{12} R_{12} Q_{1 m} Q_{2 m}=Q_{2 m} A_{12} S_{1} R_{12} .
$$

4) If $n=m=N$,

$$
R_{12} S_{1} A_{12} S_{2}=S_{2} A_{12} S_{1} R_{12}
$$

where by definition

$$
Q_{n} Q_{m}^{-1}=L_{n} B L_{n-1} B \cdots B L_{m+1} B .
$$

Proof. It consists in elementary manipulations based on Eqs. $(23,24)$ see [8]. We also use the important property that

$$
R_{12} B_{1} A_{12} B_{2}=B_{1} A_{12} B_{2} R_{12} \text {. }
$$

This is because

$$
B_{1} A_{12} B_{2}=q^{-\frac{1}{4}(H \otimes 1+1 \otimes H)^{2}}
$$

and

$$
\begin{gathered}
R_{12} \Delta_{q}(H)=\Delta_{q}^{\prime}(H) R_{12}, \\
\Delta_{q}(H)=\Delta_{q}^{\prime}(H)=H \otimes 1+1 \otimes H .
\end{gathered}
$$

To derive the relations involving $S$, we have to remember that due to the nonultralocality of relations $(23,24)$ one has

$$
L_{1 N} L_{21}=L_{21} A_{12} L_{1 N} .
$$

Finally, we will need the diagonal matrix $\varrho$ with the following properties:

$$
\begin{aligned}
\varrho_{1} \varrho_{2} & =\varrho_{2} \varrho_{1}, \\
A_{12}^{2(1-\alpha)} S_{1} \varrho_{2} A_{12}^{2 \alpha} & =\varrho_{2} S_{1}, \\
Q_{1 n} \varrho_{2} A_{12}^{2 \alpha} & =\varrho_{2} Q_{1 n} .
\end{aligned}
$$

These commutation relations are straightforward quantum generalizations of the classical formulae Eqs. $(15-17)$. We have introduced a parameter $\alpha$. It is a freedom we have also at the classical level [9]. Equations (15-17) correspond to the choice $\alpha=1 / 2$. As we will see this parameter drops out in the 
final formulae. The choice $\alpha=0$ is simpler in many respects. We give an explicit realization of $L_{n}$ and $\varrho$ in terms of a free field in the conclusion. It is not needed to derive the results of this paper.

Projecting Eq. (30) on highest weight vectors, we get

\section{Proposition 3.2. Let}

then we have.

$$
\sigma_{n}^{(j)}=\left\langle\lambda_{\max }^{(j)}\right| Q_{n}
$$

If $n \neq m$

where $\pm=\varepsilon(n-m)$.

$$
\sigma_{1 n}^{(j)} \sigma_{2 m}^{\left(j^{\prime}\right)}=\sigma_{2 m}^{\left(j^{\prime}\right)} \sigma_{1 n}^{(j)} R_{12}^{ \pm}(q)
$$

If $n=m$

$$
q^{\frac{1}{2} \lambda_{\max }^{(j)} \cdot \lambda_{\max }^{\left(j^{\prime}\right)}} \sigma_{1 n}^{(j)} \sigma_{2 n}^{\left(j^{\prime}\right)}=\sigma_{2 n}^{\left(j^{\prime}\right)} \sigma_{1 n}^{(j)} R_{12}^{+}(q)
$$

Proof. Start from Eq. (30) and use the fact that if $R_{12}=R_{12}^{+}$, then

$$
I \otimes\left\langle\lambda_{\max }^{(j)}\right| A_{12} R_{12}^{+}=I \otimes\left\langle\lambda_{\max }^{(j)}\right| .
$$

\section{Definition of the Bloch Waves}

The aim of this section is to give a precise definition of the quantum Bloch waves on the lattice. For this purpose, we will have to diagonalize the monodromy matrix Eq. (27). So let us start by giving a suitable parametrization of $S$. At the classical level, the monodromy matrix is an element of the group $\mathrm{Sl}_{2}$ and it is upper triangular. Therefore we can write

$$
S=e^{2 \pi P_{0} H} e^{Z E+} .
$$

From Eq. (22) we find that

$$
\left\{P_{0}, Z\right\}=0 \text {. }
$$

At the quantum level we look similarly for an upper triangular solution of Eq. (32) depending on two commuting operators $P_{0}$ and $Z$. The answer is

Proposition 4.1. Let $P_{0}$ and $Z$ be two commuting operators. Then the upper triangular solution of Eq. (32) is

$$
S=e^{2 \pi P_{0} H} B \sum_{i=0}^{\infty} \frac{Z^{i}}{[i] !} q^{\frac{i}{2} H} E_{+}^{i},
$$

where we used the notation

and

$$
[i]=\frac{q^{i}-q^{-i}}{q-q^{-1}}
$$

$$
[i] !=[1][2] \ldots[i]
$$

Proof. From the relations

$$
\begin{aligned}
R_{12} \Delta_{q}\left(E_{ \pm}\right) & =\Delta_{q}^{\prime}\left(E_{ \pm}\right) R_{12}, \\
\Delta_{q}\left(E_{ \pm}\right) & =E_{ \pm} \otimes q^{\frac{1}{2} H}+q^{-\frac{1}{2} H} \otimes E_{ \pm}, \\
\Delta_{q}^{\prime}\left(E_{ \pm}\right) & =q^{\frac{1}{2} H} \otimes E_{ \pm}+E_{ \pm} \otimes q^{-\frac{1}{2} H},
\end{aligned}
$$


we also have

$$
R_{12}\left[\Delta_{q}\left(E_{ \pm}\right)\right]^{i}=\left[\Delta_{q}^{\prime}\left(E_{ \pm}\right)\right]^{i} R_{12} .
$$

This gives a non-trivial identity if we insert the expansion

$$
\left[\Delta_{q}\left(E_{ \pm}\right)\right]^{i}=\sum_{j=0}^{i}\left[\begin{array}{l}
i \\
j
\end{array}\right] q^{-\frac{j}{2} H} E_{ \pm}^{i-j} \otimes q^{\frac{i-j}{2} H} E_{ \pm}^{j},
$$

where we have introduced the $q$-binomial coefficient

$$
\left[\begin{array}{l}
i \\
j
\end{array}\right]=\frac{[i] !}{[i-j] ![j] !} .
$$

Our proposition is a straightforward consequence of this identity.

Before going ahead, we give the commutation properties of the operators $P_{0}$ and $Z$ with the matrix $\varrho$. Equation (34) translates into

\section{Proposition 4.2.}

$$
\begin{gathered}
A \varrho=\varrho q^{H} A, \quad D \varrho=\varrho q^{-H} D, \\
\varrho Z=Z q^{2 \alpha H} \varrho,
\end{gathered}
$$

where we recall that $A=e^{2 \pi P_{0}}, D=e^{-2 \pi P_{0}}$.

Now that $S$ is defined, we can diagonalize it. Remembering that

$$
\sigma_{n+N}^{(j)}=\sigma_{n}^{(j)} B S
$$

we look for a matrix $g^{-1}$ such that

$$
B S g^{-1}=g^{-1} e^{2 \pi P_{0} H} B^{2} .
$$

There is a factor $B^{2}$ in the right-hand side of this equation because the diagonal part of $S$ is $e^{2 \pi P_{0} H} B$. Then

$$
\sigma_{n+N}^{(j)} g^{-1}=\sigma_{n}^{(j)} B S g^{-1}=\sigma_{n}^{(j)} g^{-1} e^{2 \pi P_{0} H} B^{2}
$$

and the vector $\sigma_{n}^{(j)} g^{-1}$ has diagonal monodromy. Of course $g^{-1}$ is determined only up to a diagonal matrix, and we choose it to be strictly upper triangular. It is interesting to have in mind the classical expression of the matrix $g^{-1}$

$$
g_{\text {clas }}^{-1}=\exp \left(-\frac{A}{A-D} Z E_{+}\right)
$$

Quantum mechanically, we have

Proposition 4.3. The solution to Eq. (39) is

$$
\begin{aligned}
g^{-1} & =\sum_{n=0}^{\infty} Z^{n} X_{n}(H) E_{+}^{n} \\
X_{n}(H) & =(-1)^{n} \frac{1}{[n] !} \frac{q^{\frac{n(n+1)}{2}} A^{n} q^{-\frac{n}{2} H}}{\prod_{v=n}^{2 n-1}\left(A q^{-H+v}-D q^{H-v}\right)}
\end{aligned}
$$


Proof. Equation (39) reads

$$
g^{-1}=e^{2 \pi P_{0} H} B^{2}\left\{\sum_{i=0}^{\infty} \frac{Z^{i}}{[i] !} q^{\frac{i}{2} H} E_{+}^{i}\right\} g^{-1} B^{-2} e^{-2 \pi P_{0} H} .
$$

Using the form Eq. (40) for $g^{-1}$, we get

$$
X_{k}(H)=e^{4 k \pi P_{0}} q^{-2 k H+2 k^{2}} \sum_{i=0}^{k} \frac{1}{[i] !} q^{\frac{i}{2} H} X_{k-i}(H-2 i) .
$$

The solution of this recursion relation is unique once we impose the boundary condition $X_{0}(H)=1$. It remains to check that Eq. (41) does satisfies this relation. Substituting Eq. (41) into Eq. (42), we arrive at $\left(x=e^{4 \pi P_{0}} q^{-2 H+2 k}\right)$

$$
\frac{1}{\prod_{v=0}^{k-1}\left(1-q^{2 v} x\right)}=\sum_{i=0}^{k}\left[\begin{array}{c}
k \\
i
\end{array}\right] \frac{x^{k-i} q^{i(k-i)}}{\prod_{v=i}^{k-1}\left(1-q^{2 v} x\right)} .
$$

This identity is easily proved by induction using Pascal's triangle identity for the $q$-binomial coefficient

$$
\left[\begin{array}{c}
k+1 \\
i
\end{array}\right]=q^{-i}\left[\begin{array}{c}
k \\
i
\end{array}\right]+q^{k+1-i}\left[\begin{array}{c}
k \\
i-1
\end{array}\right] .
$$

We are now ready to give the precise definition of the Bloch waves:

Definition. The Bloch waves are defined by

$$
\psi_{n}^{(j)}=\sigma_{n}^{(j)} g^{-1} \varrho B^{2 \alpha} .
$$

They have diagonal monodromy

$$
\psi_{n+N}^{(j)}=\psi_{n}^{(j)} e^{2 \pi P_{0} H} B^{-2} .
$$

In Eq. (44) the parameter $\alpha$ is the one appearing in Eq. $(34,35)$, and the matrix $\varrho$ satisfy the commutation properties of Proposition 4.2.

\section{Universal Bloch Wave Algebra}

Now that the Bloch waves are defined, we can compute their commutation relations. The result will be Theorem 1 of the introduction. To achieve this goal, we have to calculate the commutation relations of $\sigma_{n}^{(j)}$ with $g^{-1}$ and $\varrho$. The commutation relations of $\sigma_{n}^{(j)}$ and $\varrho$ are obtained by projecting out Eq. (35) on highest weight vectors. We get

$$
\sigma_{1 n}^{(j)} \varrho_{2} A_{12}^{2 \alpha}=\varrho_{2} \sigma_{1 n}^{(j)} .
$$

To derive those of $\sigma_{1 n}^{(j)}$ and $g^{-1}$ we first determine the commutation of $\sigma_{1 n}^{(j)}$ and $Z$. (Remember that $\sigma_{1 n}^{(j)}$ commutes with $P_{0}$.) Projecting Eq. (31), we get

$$
S_{1} \sigma_{2 n}^{(j)}=\sigma_{2 n}^{(j)} A_{12} S_{1} R_{12}^{+} \text {. }
$$


Substituting the explicit form of $S$ and $R_{12}^{+}$, we obtain

$$
Z^{k} \sigma_{n}^{(j)}=\sigma_{n}^{(j)} \sum_{i=0}^{k}\left(q-q^{-1}\right)^{i}\left[\begin{array}{c}
k \\
i
\end{array}\right] Z^{k-i} q^{-\left\{\frac{i(i+1)}{2}+i(k-i)\right\}} q^{-\left(k-\frac{i}{2}\right) H} E_{-}^{i} .
$$

For $k=1$ this gives

$$
Z \sigma_{n}^{(j)}=\sigma_{n}^{(j)}\left[Z q^{-H}+\left(1-q^{-2}\right) q^{-\frac{1}{2} H} E_{-}\right] .
$$

Of course, iterating this simple expression gives back the relation for arbitrary $k$. From these results, the commutation properties of $\sigma_{n}^{(j)}$ and $g^{-1}$ are derived by elementary calculations.

Proposition 5.1. We have

$$
g_{1}^{-1} \sigma_{2 n}^{(j)}=\sigma_{2 n}^{(j)} \mathscr{M}_{12}
$$

where

$$
\mathscr{M}_{12}=\sum_{k=0}^{\infty} \sum_{i=0}^{k}\left(q-q^{-1}\right)^{k-i}\left[\begin{array}{c}
k \\
i
\end{array}\right] Z^{i} q^{-\frac{(k-i)(k+i+1)}{2}} X_{k}\left(H_{1}\right) E_{+}^{k} \otimes q^{-\frac{k+i}{2} H} E_{-}^{k-i} .
$$

Writing

$$
\mathscr{M}_{12}=\sum_{i=0}^{\infty} Z^{i} \mathscr{M}_{12}^{(i)}
$$

one has in particular

$$
\mathscr{M}_{12}^{(0)}=\sum_{k=0}^{\infty}\left(q-q^{-1}\right)^{k} q^{-\frac{k(k+1)}{2}} X_{k}\left(H_{1}\right) E_{+}^{k} \otimes q^{-\frac{k}{2} H} E_{-}^{k} .
$$
has

Finally, let us write the commutation of $\sigma_{n}^{(j)}$ with the product $g^{-1} \varrho$. One

$$
g_{1}^{-1} \varrho_{1} \sigma_{2 m}^{(j)}=\sigma_{2 m}^{(j)} V_{12}
$$

where

$$
V_{12}=\mathscr{M}_{12} \varrho_{1} A_{12}^{2 \alpha} \text {. }
$$

We are now in a position to start the calculation of the Bloch waves algebra. We have

$$
\begin{aligned}
\psi_{1 n}^{(j)} \psi_{2 m}^{\left(j^{\prime}\right)} & =\sigma_{1 n}^{(j)} g_{1}^{-1} \varrho_{1} \sigma_{2 m}^{\left(j^{\prime}\right)} g_{2}^{-1} \varrho_{2} B_{1}^{2 \alpha} B_{2}^{2 \alpha} \\
& =\sigma_{1 n}^{(j)} \sigma_{2 m}^{\left(j^{\prime}\right)} V_{12} g_{2}^{-1} \varrho_{2} B_{1}^{2 \alpha} B_{2}^{2 \alpha} .
\end{aligned}
$$

If $n>m$ we get using Eq. (36),

$$
\psi_{1 n}^{(j)} \psi_{2 m}^{\left(j^{\prime}\right)}=\sigma_{2 m}^{\left(j^{\prime}\right)} \sigma_{1 n}^{(j)} R_{12}^{+} V_{12} g_{2}^{-1} \varrho_{2} B_{1}^{2 \alpha} B_{2}^{2 \alpha} .
$$

To reconstruct the product $\psi_{2 m}^{\left(j^{\prime}\right)} \psi_{1 n}^{(j)}$, we introduce the matrix $\mathscr{R}_{12}^{+}\left(P_{0}\right)$ such that

$$
R_{12}^{+} V_{12} g_{2}^{-1} \varrho_{2} B_{1}^{2 \alpha} B_{2}^{2 \alpha}=V_{21} g_{1}^{-1} \varrho_{1} B_{1}^{2 \alpha} B_{2}^{2 \alpha} \mathscr{R}_{12}^{+}\left(P_{0}\right)
$$

giving

$$
\psi_{1 n}^{(j)} \psi_{2 m}^{\left(j^{\prime}\right)}=\psi_{2 m}^{\left(j^{\prime}\right)} \psi_{1 n}^{(j)} \mathscr{R}_{12}^{+}\left(P_{0}\right)
$$


Notice that Eq. (47) is really a definition of the matrix $\mathscr{R}_{12}^{+}\left(P_{0}\right)$. From this equation, it depends a priori on $P_{0}$ and $Z$. The remainder of this section is devoted to the proof that $\mathscr{R}_{12}^{+}\left(P_{0}\right)$ is indeed independent of the operator $Z$. The main step will be to establish the formula

$$
V_{12} g_{2}^{-1} \varrho_{2}=\Delta_{q}\left(g^{-1}\right) \mathscr{M}_{12}^{(0)} A_{12}^{2 \alpha} \varrho_{1} \varrho_{2} .
$$

In fact, using this result, Eq. (47) becomes

$$
R_{12}^{+} \Delta_{q}\left(g^{-1}\right) \mathscr{M}_{12}^{(0)} A_{12}^{2 \alpha} \varrho_{1} \varrho_{2} B_{1}^{2 \alpha} B_{2}^{2 \alpha}=\Delta_{q}^{\prime}\left(g^{-1}\right) \mathscr{M}_{21}^{(0)} A_{12}^{2 \alpha} \varrho_{1} \varrho_{2} B_{1}^{2 \alpha} B_{2}^{2 \alpha} \mathscr{R}_{12}^{+}\left(P_{0}\right)
$$

But

$$
R_{12}^{+} \Delta_{q}\left(g^{-1}\right)=\Delta_{q}^{\prime}\left(g^{-1}\right) R_{12}^{+},
$$

and we can eliminate the factor $\Delta_{q}^{\prime}\left(g^{-1}\right)$ which contains actually all the $Z$ dependence. Commuting the factors $A_{12}^{2 \alpha} B_{1}^{2 \alpha} B_{2}^{2 \alpha}$ and $\varrho_{1} \varrho_{2}$, we are left with the universal formula for $\mathscr{R}_{12}^{+}\left(P_{0}\right)$ given in the introduction with

$$
F_{12}\left(q, P_{0}\right)=\mathscr{M}_{12}^{(0)}\left(A q^{H_{1}+H_{2}}, D q^{-H_{1}-H_{2}}\right) .
$$

We now turn to the proof of Eq. (48). One has to study

$$
V_{12} g_{2}^{-1} \varrho_{2}=\mathscr{M}_{12} \varrho_{1} A_{12}^{2 \alpha} g_{2}^{-1} \varrho_{2} \text {. }
$$

First we can push the factor $\varrho_{1} A_{12}^{2 \alpha}$ to the right. We find

$$
\varrho_{1} A_{12}^{2 \alpha} g_{2}^{-1} \varrho_{2}=\left(\sum_{i=0}^{\infty} Z^{i} X_{i}\left(H_{1}+H_{2}\right) q^{-\frac{i}{2} H} \otimes E_{+}^{i}\right) A_{12}^{2 \alpha} \varrho_{1} \varrho_{2} .
$$

Next we make the $Z$-dependence explicit by writing

where

$$
V_{12} g_{2}^{-1} \varrho_{2}=\left(\sum_{i=0}^{\infty} Z^{i} \mathscr{V}_{12}^{(i)}\right) A_{12}^{2 \alpha} \varrho_{1} \varrho_{2}
$$

$$
\mathscr{V}_{12}^{(i)}=\sum_{j=0}^{i} X_{i-j}\left(H_{1}+H_{2}-2 j\right) \mathscr{M}_{12}^{(j)} q^{-\frac{i-j}{2} H} \otimes E_{+}^{i-j} .
$$

We now have to commute the factors $E_{+}$to the left. This is done with the help of the identity

$$
\begin{aligned}
& \mathscr{M}_{12}^{(j)} q^{-\frac{1}{2} H} \otimes E_{+}=q^{-j}\left\{E_{+} \otimes q^{\frac{1}{2} H}+q^{-\frac{1}{2} H} \otimes E_{+}\right\} \mathscr{M}_{12}^{(j)} \\
& \quad+q^{-j-1}[j+1] D q^{\frac{1}{2}\left(H_{1}+H_{2}\right)}\left\{A q^{-H_{1}-H_{2}+j+1}-D q^{H_{1}+H_{2}-j-1}\right\} \mathscr{M}_{12}^{(j+1)},
\end{aligned}
$$

the proof of which is elementary and uses the relation

$$
X_{k-1}(H-2)=-[k]\left(A q^{-H+k}-D q^{H-k}\right) D q^{\frac{1}{2} H-1} X_{k}(H) .
$$

We can iterate the above identity and get

$$
\mathscr{M}_{12}^{(j)}\left[q^{-\frac{1}{2} H} \otimes E_{+}\right]^{k}=\sum_{l=0}^{k}\left[\Delta_{q}\left(E_{+}\right)\right]^{k-l} C_{l}^{j, k}\left(H_{1}+H_{2}\right) \mathscr{M}_{12}^{(j+l)},
$$

where the coefficients $C_{l}^{j, k}(x)$ satisfy the recursion relation

$$
\begin{aligned}
C_{l}^{j, k+1}(x)= & q^{-j-l} C_{l}^{j, k}(x+2) \\
& +q^{-j-l}[j+l] D q^{\frac{1}{2} x} C_{l-1}^{j, k}(x)\left(A q^{-x+j+l}-D q^{x-j-l}\right)
\end{aligned}
$$


whose solution is

$$
C_{l}^{j, k}(x)=q^{\frac{l}{2} x} D^{l} q^{-k j-\frac{l(l+1)}{2}} \frac{[j+l] !}{[j] !}\left[\begin{array}{l}
k \\
l
\end{array}\right]_{v=j-k+l+1}^{j-k+2 l}\left(A q^{-x+v}-D q^{x-v}\right) .
$$

Using this result, we can write $\left(x=H_{1}+H_{2}\right)$.

$$
\mathscr{V}_{12}^{(i)}=\sum_{r=0}^{i}\left\{\sum_{j=0}^{r} X_{i-j}(x-2 j) C_{r-j}^{j, i-j}(x-2 i+2 r)\right\}\left[\Delta_{q}\left(E_{+}\right)\right]^{i-r} \mathscr{M}_{12}^{(r)} .
$$

For $r=0$, the sum over $j$ is simply equal to $X_{i}(x)$. For $r=1$ we easily check that it vanishes. We assume now $r \geqq 2$. One has $\left(z=A^{2} q^{-2 x}\right)$

$$
\begin{aligned}
& X_{i-j}(x-2 j) C_{r-j}^{j, i-j}(x-2 i+2 r)=X_{i}(x)\left(1-z q^{2 i}\right) \\
& \cdot(-1)^{r} q^{-\frac{r}{2} x} z^{-r} q^{-r(2 i-r+1)} \frac{[i] !}{[i-r] !}(-1)^{j} q^{-j(r-1)}\left[\begin{array}{l}
r \\
j
\end{array}\right] \prod_{v=i+j-r+1}^{i+j-1}\left(1-z q^{2 v}\right) .
\end{aligned}
$$

So the sum over $j$ in Eq. (51) reduces to the evaluation of

$$
\sum_{j=0}^{r}(-1)^{j} q^{-j(r-1)}\left[\begin{array}{l}
r \\
j
\end{array}\right]_{\nu=i+j-r+1}^{i+j-1}\left(1-z q^{2 \nu}\right) .
$$

Expanding the product by means of the $q$-binomial formula

$$
\prod_{v=0}^{n-1}\left(1-z q^{2 v}\right)=\sum_{v=0}^{n}(-1)^{v}\left[\begin{array}{l}
n \\
v
\end{array}\right] q^{(n-1) v} z^{v},
$$

expression Eq. (52) becomes

$$
\sum_{v=0}^{r-2}(-1)^{v}\left[\begin{array}{c}
r-1 \\
v
\end{array}\right] q^{v(2 i-r)} z^{\nu} \sum_{j=0}^{r}(-1)^{j}\left[\begin{array}{l}
r \\
j
\end{array}\right] q^{j(2 v-r+1)},
$$

but we have, again using Eq. (53)

$$
\sum_{j=0}^{r}(-1)^{j}\left[\begin{array}{l}
r \\
j
\end{array}\right] q^{j(r-1)}\left[q^{2(v-r+1)}\right]^{j}=\prod_{\mu=0}^{r-1}\left(1-q^{2 \mu} q^{2(v-r+1)}\right)
$$

which is zero for $v=0,1 \ldots(r-1)$. Therefore, only the term $r=0$ remains in Eq. (51) and we finally get

$$
\mathscr{V}_{12}^{(i)}=X_{i}\left(H_{1}+H_{2}\right)\left[\Delta_{q}\left(E_{+}\right)\right]^{i} \mathscr{M}_{12}^{(0)} .
$$

This is exactly what we had to prove.

\section{The $\xi$-Basis}

Since the Bloch waves are now well under control, we may try to construct other basis from this one. In particular we can try to find a basis $\xi$ with the exchange algebra given by Eq. (9). We have already defined the vectors $\sigma^{(j)}(x)$ with this property, however since $Q(x)$ is normalized to one at $x=0$, they do not have good conformal properties (in the continuum limit). We have shown in [6] that for $j=1 / 2$ there exists a change of basis depending only on $P_{0}$

$$
\xi^{(j)}(x)=\psi^{(j)}(x) M\left(P_{0}\right)
$$


such that $\xi^{(1 / 2)}(x)$ does satisfy Eq. (9). This result was later generalized to all $j$ in [13]. We give here the universal form of $M\left(P_{0}\right)$. Since $M$ depends only on $P_{0}$ and since $P_{0}$ commutes with the Virasoro generators, $\xi^{(j)}(x)$ has the same good conformal properties as $\psi^{(j)}(x)$.

Before studying the quantum case, it is worth giving the form of the matrix $M\left(P_{0}\right)$ in the classical case. Remembering that

$$
\left\{\psi^{(j)}(x), P_{0}\right\}=\frac{\gamma}{2 \pi} \psi^{(j)}(x) H
$$

the requirement that $\psi^{(j)}(x)$ satisfies Eq. (5) gives the condition on $M$

$$
\begin{aligned}
r^{+} & +r^{-}+\frac{A+D}{A-D} M^{-1} \otimes M^{-1}\left(r^{+}+r^{-}\right) M \otimes M \\
& =\frac{2}{\pi}\left[M^{-1} \frac{d M}{d P_{0}} \otimes M^{-1} H M-M^{-1} H M \otimes M^{-1} \frac{d M}{d P_{0}}\right] .
\end{aligned}
$$

We look for $M$ in the form

$$
M=e^{m_{+}\left(P_{0}\right) E_{+}} e^{m_{-}\left(P_{0}\right) E_{-}} .
$$

Using that

$$
\operatorname{ad}\left(E_{ \pm} \otimes I+I \otimes E_{ \pm}\right) r=2\left(E_{ \pm} \otimes H-H \otimes E_{ \pm}\right),
$$

we find the relations

$$
\frac{1}{2 \pi} m_{+}^{\prime}=-\frac{A+D}{A-D} m_{+}, \quad \frac{1}{2 \pi} m_{-}^{\prime}=m_{-}, \quad \frac{1}{2 \pi} m_{+} m_{-}^{\prime}=-\frac{A}{A-D} .
$$

So that

$$
M_{\text {clas }}\left(P_{0}\right)=\exp \left(\frac{1}{A-D} E_{+}\right) \exp \left(-A E_{-}\right) .
$$

At the quantum level, the equation one has to solve reads (we write $M(A, D)$ instead of $M\left(P_{0}\right)$ )

$$
\begin{aligned}
& R_{12}^{ \pm} F_{12} M_{1}\left(A q^{H_{2}}, D q^{-H_{2}}\right) M_{2}(A, D) \\
& \quad=F_{21} M_{2}\left(A q^{H_{1}}, D q^{-H_{1}}\right) M_{1}(A, D) R_{12}^{ \pm} .
\end{aligned}
$$

Its solution is given in Theorem 2 of the introduction. This theorem is itself a direct consequence of the following

Proposition 6.1. One has

$$
F_{12} M_{1}\left(A q^{H_{2}}, D q^{-H_{2}}\right) M_{2}(A, D)=\Delta_{q}[M(A, D)] .
$$

Proof. The crucial identity is

$$
\begin{aligned}
F_{12}\left[q^{-\frac{1}{2} H} \otimes E_{+}\right]^{k}= & \sum_{l=0}^{k}\left[\Delta_{q}\left(E_{+}\right)\right]^{k-l} \\
& \cdot C_{l}^{0, k}\left(H_{1}+H_{2}, A q^{H_{1}+H_{2}+2(k-l)}, D q^{-H_{1}-H_{2}-2(k-l)}\right) \\
& \cdot \mathscr{M}_{12}^{(l)}\left(A q^{H_{1}+H_{2}+2(k-l)}, D q^{-H_{1}-H_{2}-2(k-l)}\right) .
\end{aligned}
$$


It is obtained by multiplying Eq. (50) on the left by $\varrho_{1} \varrho_{2}$ and then moving this factor to the right. With the help of this identity, we get

$$
\begin{aligned}
F_{12} & M_{1}\left(A q^{H_{2}}, D q^{-H_{2}}\right) M_{2}(A, D) \\
= & \sum_{n, b, m, j}\left[\Delta_{q}\left(E_{+}\right)\right]^{n} E_{+}^{b}\left\{\left[\begin{array}{c}
m \\
j
\end{array}\right] q^{-\frac{j}{2} H} E_{-}^{m-j} \otimes q^{\frac{m-j}{2} H} E_{-}^{j}\right\} \\
& \cdot q^{\frac{b}{2} H_{1}+n b+\frac{1}{2} b(b-1)+b(m-j)} \mathscr{T}_{b}\left(H_{2}+2(n-j)\right) \Delta_{q}\left[M_{n m}(H)\right],
\end{aligned}
$$

where the coefficients $M_{n m}(H)$ are those entering the definition of $M\left(P_{0}\right)$

$$
M\left(P_{0}\right)=\sum_{n, m=0}^{\infty} E_{+}^{n} E_{-}^{m} M_{n m}(H)
$$

and

$$
\begin{aligned}
\mathscr{T}_{b}(x)= & \sum_{s}\left\{\sum_{r}(-1)^{r}\left(q-q^{-1}\right)^{r}\left[\begin{array}{l}
j \\
r
\end{array}\right] \frac{q^{\frac{1}{2} r(r+1)-r(s+j)}}{[s-r] !}\right\} \\
& \cdot \frac{(-1)^{s}}{[b-s] !} q^{2 s j} \frac{A q^{x+2 s}-D q^{-x-2 s}}{\prod_{v=s}^{b+s}\left(A q^{x+v}-D q^{-x-v}\right)} .
\end{aligned}
$$

We use

$$
\left[\begin{array}{l}
n \\
p
\end{array}\right]=\frac{(-1)^{p}}{[p] !\left(q-q^{-1}\right)^{p}} q^{-n p+\frac{1}{2} p(p-1)} \sum_{v=0}^{p}(-1)^{v}\left[\begin{array}{l}
p \\
v
\end{array}\right] q^{(p-1) v} q^{2(n-p+1) v}
$$

and the analog formula with $q \rightarrow q^{-1}$ to show that

$$
\sum_{r}(-1)^{r}\left(q-q^{-1}\right)^{r}\left[\begin{array}{l}
j \\
r
\end{array}\right] \frac{q^{\frac{1}{2} r(r+1)-r(s+j)}}{[s-r] !}=\frac{1}{[s] !} q^{-2 s j} .
$$

So

$$
\mathscr{T}_{b}(x)=\sum_{s} \frac{(-1)^{s}}{[s] ![b-s] !} \frac{A q^{x+2 s}-D q^{-x-2 s}}{\prod_{v=s}^{b+s}\left(A q^{x+v}-D q^{-x-v}\right)} .
$$

Now, the proposition results from the fact that

$$
\mathscr{T}_{b}(x)=\delta_{b, 0} .
$$

Indeed, if $b=0$ then $s=0$ and $\mathscr{T}_{0}(x)=1$. let us assume $b>0$. One has to prove that $\left(z=A^{2} q^{2 x}\right)$

$$
\sum_{s=0}^{b}(-1)^{s}\left[\begin{array}{l}
b \\
s
\end{array}\right] q^{(b-1) s}\left(1-z q^{4 s}\right) \prod_{v=0}^{s-1}\left(1-z q^{2 v}\right) \prod_{v=b+s+1}^{2 b}\left(1-z q^{2 v}\right)=0 \quad \forall z .
$$

Using the binomial formula Eq. (53) we obtain

$$
\begin{aligned}
(1- & \left.z q^{4 s}\right) \prod_{v=0}^{s-1}\left(1-z q^{2 v}\right) \prod_{v=b+s+1}^{2 b}\left(1-z q^{2 v}\right) \\
& =\sum_{\mu=0}^{b+1}(-1)^{\mu} z^{\mu} \sum_{v}\left\{F(\mu, v, s)+q^{4 s} F(\mu-1, v, s)\right\},
\end{aligned}
$$


where

$$
F(\mu, v, s)=q^{(3 b+1) \mu} q^{s \mu-(3 b+2) v}\left[\begin{array}{l}
s \\
v
\end{array}\right]\left[\begin{array}{l}
b-s \\
\mu-v
\end{array}\right] .
$$

To perform the summation over $v$ in Eq. (57) we substitute for the $q$-binomial coefficients the identity Eq. (55). We get

$$
\begin{aligned}
\sum_{v} F(\mu, v, s)= & q^{2 b \mu} \sum_{m}\left(q-q^{-1}\right)^{-m} q^{2 s m} \frac{q^{-\frac{1}{2} m(m+1)-2 b m}}{[m] !}\left[\begin{array}{c}
2 b+1 \\
\mu-m
\end{array}\right] \\
& \cdot \sum_{v^{\prime}}(-1)^{v^{\prime}}\left[\begin{array}{c}
m \\
v^{\prime}
\end{array}\right] q^{(m-1) v^{\prime}} q^{2(b+1) v^{\prime}} .
\end{aligned}
$$

The summation over $v^{\prime}$ can now be done using again Eq. (55)

$$
\sum_{v} F(\mu, v, s)=q^{2 b \mu} \sum_{m=0}^{\mu}(-1)^{m} q^{2 s m} q^{-b m}\left[\begin{array}{c}
b+m \\
b
\end{array}\right]\left[\begin{array}{c}
2 b+1 \\
\mu-m
\end{array}\right] .
$$

Substituting back into Eq. (56) the summation over $s$ can be done, again using Eq. (55)

$$
\begin{gathered}
\sum_{s=0}^{b}(-1)^{s}\left[\begin{array}{l}
b \\
s
\end{array}\right] q^{(b-1) s} \sum_{v} F(\mu, v, s)=(-1)^{b}[b] !\left(q-q^{-1}\right)^{b} q^{\frac{1}{2} b(b-1)+2 b \mu} \\
\cdot \sum_{m}(-1)^{m}\left[\begin{array}{c}
b+m-1 \\
b
\end{array}\right]\left[\begin{array}{c}
b+m \\
b
\end{array}\right]\left[\begin{array}{c}
2 b+1 \\
\mu-m
\end{array}\right]
\end{gathered}
$$

Similarly, we have

$$
\begin{aligned}
& \sum_{s=0}^{b}(-1)^{s}\left[\begin{array}{l}
b \\
s
\end{array}\right] q^{(b-1) s} q^{4 s} \sum_{v} F(\mu-1, v, s)=(-1)^{b}[b] !\left(q-q^{-1}\right)^{b} q^{\frac{1}{2} b(b-1)+2 b \mu} \\
& \cdot \sum_{m}(-1)^{m}\left[\begin{array}{c}
b+m+1 \\
b
\end{array}\right]\left[\begin{array}{c}
b+m \\
b
\end{array}\right]\left[\begin{array}{c}
2 b+1 \\
\mu-m-1
\end{array}\right] .
\end{aligned}
$$

Adding these two expressions as required in Eq. (56), the terms cancel two by two.

\section{The Second Chirality}

In this section we define the various objects entering the second chiral sector, and we list their basic relations. One can then reproduce the previous analysis. The results are summarised in Theorem 3.

The Drinfeld-Sokolov linear system for the second chirality reads [9]

$$
\partial \bar{Q}=-\bar{Q}\left(\bar{P} H-E_{-}\right) \text {. }
$$

Poisson bracket is defined by

$$
\{\bar{P}(x) \stackrel{\otimes}{, P}(y)\}=-\gamma \delta^{\prime}(x-y) .
$$

We discretize Eq. (58) as

$$
\bar{Q}_{n}=\bar{Q}_{n-1} \bar{L}_{n}
$$


On $\bar{L}_{n}$ we have the Poisson bracket

$$
\begin{aligned}
\left\{\bar{L}_{n} \otimes \bar{L}_{m}\right\}= & -\frac{\gamma}{2} \delta_{n, m}\left[r, \bar{L}_{n} \otimes \bar{L}_{m}\right] \\
& +\frac{\gamma}{2} \delta_{n, m+1} 1 \otimes \bar{L}_{m}(H \otimes H) \bar{L}_{n} \otimes 1 \\
& -\frac{\gamma}{2} \delta_{n, m-1} \bar{L}_{n} \otimes 1(H \otimes H) 1 \otimes \bar{L}_{m} .
\end{aligned}
$$

Performing the replacement

$$
[,]=i\{,\}
$$

we find the quantum formulae

$$
\begin{aligned}
\bar{R}_{12} \bar{L}_{1 n} \bar{L}_{2 n} & =\bar{L}_{2 n} \bar{L}_{1 n} \bar{R}_{12}, \\
\bar{L}_{1, n+1} \bar{L}_{2 n} & =\bar{L}_{2 n} \bar{A}_{12} \bar{L}_{1, n+1}
\end{aligned}
$$

with

$$
\begin{aligned}
& \bar{R}_{12} \rightarrow 1+\frac{i \gamma}{2} r+O\left(\gamma^{2}\right), \\
& \bar{A}_{12} \rightarrow 1+\frac{i \gamma}{2} H \otimes H+O\left(\gamma^{2}\right) .
\end{aligned}
$$

So

$$
\begin{aligned}
& \bar{A}_{12}=A_{12}, \\
& \bar{R}_{12}=R_{12}\left(q^{-1}\right)=R_{12}^{-1}(q) .
\end{aligned}
$$

Finally, we introduce the constant $\bar{\varrho}$ with the properties

$$
\begin{aligned}
\bar{\varrho}_{1} \bar{\varrho}_{2} & =\bar{\varrho}_{2} \bar{\varrho}_{1}, \\
\bar{Q}_{1 n} \bar{\varrho}_{2} & =A_{12}^{2 \bar{\alpha}} \bar{\varrho}_{2} \bar{Q}_{1 n}, \\
\bar{S}_{1} \bar{\varrho}_{2} & =A_{12}^{2 \bar{\alpha}} \bar{\varrho}_{2} \bar{S}_{1} A_{12}^{2(1-\bar{\alpha})} .
\end{aligned}
$$

We consider now a periodic lattice with $N$ sites and define

$$
\begin{aligned}
\bar{Q}_{n} & =\bar{L}_{1} B \bar{L}_{2} B \cdots B \bar{L}_{n}, \\
\bar{S} & =\bar{L}_{1} B \bar{L}_{2} \cdots B \bar{L}_{N},
\end{aligned}
$$

so that

$$
\bar{Q}_{n+N}=\bar{S} B \bar{Q}_{n} .
$$

We have

Proposition 7.1. 1) If $n<N$

$$
\bar{R}_{12} \bar{Q}_{1 n} \bar{Q}_{2 n}=\bar{Q}_{2 n} \bar{Q}_{1 n} \bar{R}_{12} .
$$

2) If $n>m(n, m<N)$

$$
\bar{Q}_{2 m} \bar{Q}_{1 m} \bar{R}_{12} A_{12} \bar{Q}_{1 m}^{-1} \bar{Q}_{1 n}=\bar{R}_{12} \bar{Q}_{1 n} \bar{Q}_{2 m} .
$$

3) If $m=N, n<N$

$$
\bar{Q}_{2 n} \bar{Q}_{1 n} \bar{R}_{12} A_{12} \bar{Q}_{1 n}^{-1} \bar{S}_{1}=\bar{R}_{12} \bar{S}_{1} A_{12} \bar{Q}_{2 n} .
$$

4) If $n=m=N$

$$
\bar{R}_{12} \bar{S}_{1} A_{12} \bar{S}_{2}=\bar{S}_{2} A_{12} \bar{S}_{1} \bar{R}_{12}
$$


We now define the projection on the first column of $\bar{Q}_{n}$

$$
\bar{\sigma}_{n}^{(j)}=\bar{Q}_{n}\left|\lambda_{\max }^{(j)}\right\rangle
$$

then we have the exchange algebra

$$
\bar{\sigma}_{1 n}^{(j)} \bar{\sigma}_{2 m}^{\left(j^{\prime}\right)}=R_{12}^{\mp}(q) \bar{\sigma}_{2 m}^{\left(j^{\prime}\right)} \bar{\sigma}_{1 n}^{(j)},
$$

where the upper sign refers to $n>m$ and the lower one to $n<m$.

We parametrize the solution of Eq. (62) as

$$
\bar{S}=e^{-2 \pi \bar{p}_{0} H} B \sum_{i=0}^{\infty} \frac{\bar{Z}^{i}}{[i] !} q^{-\frac{i}{2} H} E_{-}^{i} .
$$

The equation determining $\bar{g}$ is

$$
\bar{g} \bar{S} B=e^{-2 \pi \bar{P}_{0} H} B^{2} \bar{g} .
$$

The Bloch waves are defined as

$$
\bar{\psi}_{n}^{(j)}=B^{2 \bar{\alpha}} \bar{\varrho} \bar{g} \bar{\sigma}_{n}^{(j)} .
$$

They have diagonal monodromy

$$
\bar{\psi}_{n+N}^{(j)}=B^{-2} e^{-2 \pi \bar{P}_{0} H} \bar{\psi}_{n}^{(j)} .
$$

Reproducing the analysis from these data we arrive at the

\section{Theorem 3.}

$$
\begin{aligned}
\bar{\psi}_{1 n}^{(j)} \bar{\psi}_{2 m}^{\left(j^{\prime}\right)} & =\overline{\mathscr{R}}_{12}^{ \pm}\left(q, \bar{P}_{0}\right) \bar{\psi}_{2 m}^{\left(j^{\prime}\right)} \bar{\psi}_{1 n}^{(j)}, \\
\overline{\mathscr{R}}_{12}^{ \pm}\left(q, \bar{P}_{0}\right) & =\bar{F}_{12}\left(q, \bar{P}_{0}\right) R_{12}^{\mp}(q) \bar{F}_{21}^{-1}\left(q, \bar{P}_{0}\right),
\end{aligned}
$$

where

$$
\begin{aligned}
& \bar{F}_{12}\left(q, \bar{P}_{0}\right) \\
& \quad=\sum_{k=0}^{\infty}\left(q-q^{-1}\right)^{k} \frac{(-1)^{k}}{[k] !} \frac{\bar{A}^{k}}{\prod_{v=1}^{k}\left(\bar{A} q^{H_{1}-v}-\bar{D} q^{-H_{1}+v}\right)} q^{\frac{k}{2}\left(H_{1}+H_{2}\right)} E_{+}^{k} \otimes E_{-}^{k}
\end{aligned}
$$

and

$$
\bar{A}=e^{-2 \pi \bar{P}_{0}}, \quad \bar{D}=e^{2 \pi \bar{P}_{0}} .
$$

\section{Local Fields}

In this section we combine the two chiralities to construct a set of periodic and local fields. In order to do it we have to impose the constraint $P_{0}=\bar{P}_{0}$. We choose to work with independent zero modes $P_{0}$ and $\bar{P}_{0}$. Their conjugate variables are also considered to be independent so that $\psi^{(j)}(x)$ and $\bar{\psi}^{\left(j^{\prime}\right)}(x)$ commute

$$
\psi_{1}^{(j)}(x) \bar{\psi}_{2}^{\left(j^{\prime}\right)}(y)=\bar{\psi}_{2}^{\left(j^{\prime}\right)}(y) \psi_{1}^{(j)}(x)
$$


The constraint $P_{0}=\bar{P}_{0}$ is then imposed on the states. If $\mathscr{H}$ and $\overline{\mathscr{H}}$ are the representation spaces of the two chiral halves of the theory, the physical space $\mathscr{F} \subset \mathscr{H} \otimes \overline{\mathscr{H}}$ is defined by the condition

$$
\left(P_{0}-\bar{P}_{0}\right) \mathscr{F}=0,
$$

i.e. we have the decomposition

$$
\mathscr{F}=\underset{j}{\oplus} \mathscr{H}_{\omega_{j}} \otimes \overline{\mathscr{H}}_{\omega_{j}}
$$

where $\omega_{j}$ denote the eigenvalues of $P_{0}$. The result is summarized in the

Theorem 4. The fields

$$
\Phi_{n}^{(j)}=\psi_{n}^{(j)} B^{-1} \bar{\psi}_{n}^{(j)} .
$$

1) Admit a restriction to the subspace $\mathscr{F}$, where $P_{0}=\bar{P}_{0}$ or

$$
(A \bar{A}-D \bar{D}) \mathscr{F}=0 .
$$

2) Their restriction to $\mathscr{F}$ is periodic.

3) Their restriction to $\mathscr{F}$ is local.

Proof. 1) Recall that

$$
\begin{array}{ll}
\psi_{n}^{(j)} A=A \psi_{n}^{(j)} q^{-H}, & \psi_{n}^{(j)} D=D \psi_{n}^{(j)} q^{H} \\
\bar{\psi}_{n}^{(j)} \bar{A}=\bar{A} q^{H} \bar{\psi}_{n}^{(j)}, & \bar{\psi}_{n}^{(j)} \bar{D}=\bar{D} q^{-H} \bar{\psi}_{n}^{(j)}
\end{array}
$$

then

$$
\Phi_{n}^{(j)}(A \bar{A}-D \bar{D})=(A \bar{A}-D \bar{D}) \Phi_{n}^{(j)},
$$

and so

$$
(A \bar{A}-D \bar{D}) \Phi_{n}^{(j)} \mathscr{F}=\Phi_{n}^{(j)}(A \bar{A}-D \bar{D}) \mathscr{F}=0,
$$

therefore

$$
\Phi_{n}^{(j)} \mathscr{F} \subset \mathscr{F} .
$$

2) We have

$$
\begin{aligned}
\Phi_{n+N}^{(j)} & =\psi_{n+N}^{(j)} B^{-1} \psi_{n+N}^{(j)} \\
& =\psi_{n}^{(j)} e^{2 \pi P_{0} H} B^{-2} B^{-1} e^{-2 \pi \bar{P}_{0} H} B^{-2} \bar{\psi}_{n}^{(j)} \\
& =\psi_{n}^{(j)} B^{-1} B^{-4} e^{2 \pi\left(P_{0}-\bar{P}_{0}\right) H} \bar{\varrho} \bar{g} \bar{\sigma}_{n}^{(j)}
\end{aligned}
$$

but

$$
B^{-4} e^{2 \pi\left(P_{0}-\bar{P}_{0}\right) H} \bar{\varrho}=\varrho \varrho e^{2 \pi\left(P_{0}-\bar{P}_{0}\right) H} .
$$

So

$$
\Phi_{n+N}^{(j)}=\psi_{n+N}^{(j)} B^{-1} \varrho e^{\left(P_{0}-\bar{P}_{0}\right) H} \bar{g} \bar{\sigma}_{n}^{(j)} .
$$

Now $\bar{g}$ and $\bar{\sigma}_{n}^{(j)}$ do not contain the variables conjugate to $P_{0}$ or $\bar{P}_{0}$. So when restricting to $\mathscr{F}$ we can set $P_{0}=\bar{P}_{0}$ in Eq. (63) and

$$
\left.\Phi_{n+N}^{(j)}\right|_{\mathscr{F}}=\left.\Phi_{n}^{(j)}\right|_{\mathscr{F}} \text {. }
$$


3) Let us prove now locality. We calculate $(n>m)$

$$
\left[\Phi_{n}^{(j)}, \Phi_{m}^{\left(j^{\prime}\right)}\right]=\psi_{2 m}^{\left(j^{\prime}\right)} \psi_{1 n}^{(j)}\left(\mathscr{R}_{12}^{+} B_{1}^{-1} B_{2}^{-1} \overline{\mathscr{R}}_{12}^{+}-B_{1}^{-1} B_{2}^{-1}\right) \bar{\psi}_{2 m}^{\left(j^{\prime}\right)} \bar{\psi}_{1 n}^{(j)}
$$

but

$$
\mathscr{R}_{12}^{+} B_{1}^{-1} B_{2}^{-1} \overline{\mathscr{R}}_{12}^{+}=F_{21}^{-1} R_{12}^{+} F_{12} A_{12} \bar{F}_{12} R_{12}^{-} \bar{F}_{21}^{-1} A_{12}^{-1} B_{1}^{-1} B_{2}^{-1} .
$$

If we can show that

$$
\begin{aligned}
R_{12}^{+} F_{12} A_{12} \bar{F}_{12} & =1+X_{12} \cdot\left(A \bar{A} q^{H_{1}+H_{2}}-D \bar{D} q^{-H_{1}-H_{2}}\right), \\
F_{21}^{-1} R_{12}^{-} \bar{F}_{21}^{-1} A_{12}^{-1} & =1+\bar{X}_{12} \cdot\left(A \bar{A} q^{H_{1}+H_{2}}-D \bar{D} q^{-H_{1}-H_{2}}\right),
\end{aligned}
$$

then

$$
\begin{aligned}
{\left[\Phi_{n}^{(j)}, \Phi_{m}^{\left(j^{\prime}\right)}\right]=} & \psi_{2 m}^{\left(j^{\prime}\right)} \psi_{1 n}^{(j)}\left(\bar{X}_{12}+F_{21}^{-1} X_{12} R_{12}^{-} \bar{F}_{21}^{-1} A_{12}^{-1}\right) B_{1}^{-1} B_{2}^{-1} \bar{\psi}_{2 m}^{\left(j^{\prime}\right)} \bar{\psi}_{1 n}^{(j)} \\
& \cdot(A \bar{A}-D \bar{D})
\end{aligned}
$$

and this implies the desired result

$$
\left[\Phi_{n}^{(j)}, \Phi_{m}^{\left(j^{\prime}\right)}\right] \mathscr{F}=0 .
$$

We observe now that condition Eq. (65) is the same as condition Eq. (64). Indeed Eq. (65) is equivalent to

$$
R_{21}^{+} F_{21} A_{12} \bar{F}_{21}=1-R_{21}^{+} F_{21} \bar{X}_{12} A_{12} \bar{F}_{21}\left(A \bar{A} q^{H_{1}+H_{2}}-D \bar{D} q^{-H_{1}-H_{2}}\right) .
$$

Comparing with Eq. (64) we find the relation

$$
\bar{X}_{12}=-F_{21}^{-1} R_{12}^{-} X_{21} \bar{F}_{21}^{-1} A_{12}^{-1} \text {. }
$$

To prove Eq. (64) we evaluate its left-hand side

$$
\begin{aligned}
R_{12}^{+} F_{12} A_{12} \bar{F}_{12}= & \sum_{k=0}^{\infty}\left(q-q^{-1}\right)^{k} \frac{1}{[k] !} q^{-\frac{1}{2} k(k+1)} \\
& \cdot\left\{\sum_{i=0}^{k}\left[\begin{array}{c}
k \\
i
\end{array}\right] q^{-i(k-i)+2 i^{2}} \frac{1}{\prod_{v=i}^{i-1}\left(1-z q^{2 v}\right)} \frac{(\bar{z})^{k-i}}{\prod_{v=2 i+1}^{k+i}\left(1-\bar{z} q^{-2 v}\right)}\right\} \\
& \cdot q^{-\frac{k}{2}\left(H_{1}-H_{2}\right)} E_{+}^{k} \otimes E_{-}^{k},
\end{aligned}
$$

where we have introduced the notation

$$
z=A^{2} q^{2 H_{2}}, \quad \bar{z}=\bar{A}^{2} q^{2 H_{1}} .
$$

We are thus lead to study the quantity in the curly bracket. We have to show that this expression contains a factor $(1-z \bar{z})$ (for $k>0)$, or else that it vanishes if we set $\bar{z}=1 / z$. This conditon turns out to be equivalent to Eq. (56).

\section{Conclusion}

We would like to conclude this paper with a few remarks. The various formulae we have presented here are universal in the sense that they are expressed entirely in terms of the generators $H, E_{ \pm}$of $\mathscr{U}_{q}\left(s l_{2}\right)$. This means that we have been able to use the quantum group structure of the theory in its full power. 
In particular, we have been able to separate in our formulae the purely group theoretical aspects from the representation theoretic aspects. This may be of some interest as we may consider now highest weight representations with negative spin $j$ as it seems to be necessary in Liouville theory or $2 D$ gravity [14].

The main tool to achieve these results was a lattice version of the Drinfeld-Sokolov linear system. This is by itself an interesting structure. One can eventually use it to define a lattice version of the Coulomb gas picture of conformal field theory. This is based on the following free field representation of the basic relations Eqs. $(23,24)$. Let $\varphi_{n}$ be a periodic free field on the lattice and $\pi_{n}$ its conjugate momentum so that

$$
\left[\pi_{n}, \varphi_{m}\right]=i \gamma \frac{\delta_{n m}}{a},
$$

where $a$ is the lattice spacing. A realization of Eqs. $(23,24)$ is given by

$$
L_{n}=e^{a \pi_{n} H}\left\{B \sum_{i=0}^{\infty} \frac{a^{i}}{[i] !} q^{\frac{i}{2} H} E_{+}^{i}\right\} e^{\frac{1}{2}\left(\varphi_{n-1}-\varphi_{n}\right) H} .
$$

Moreover the constant $\varrho$ satisfying Eqs. (33-35) (with $\alpha=0$ ) is

$$
\varrho=e^{-\varphi_{N} H} .
$$

We will analyse this interesting representation somewhere else.

A natural question which arises is: What happens to the Virasoro algebra? Clearly, in the continuous theory, one can reconstruct the Virasoro generators from the $\xi$ or the $\psi$ fields. The same is true on the lattice and we get an interesting lattice deformation of the Virasoro algebra $[15-17,19]$. Let us simply mention here that this deformation is related to integrable systems (Toda chain) [18].

Finally, it is well known that the Drinfeld-Sokolov linear system is a reduction of a more general system which we would obtain if we were working with the WZNW model instead of the Liouville model. A discrete version of the WZNW model was recently proposed [20]. It is likely to be closely related to our construction.

Acknowledgements. I am very grateful to L. Bonora and F. Toppan for their encouragements and help at various stages of this work.

\section{References}

1. Poincaré, H.: Les fonctions fuchsiennes et l'équation $\Delta u=e^{u}$. J. Math. 4(5), 137 (1898)

2. Hawley, N.S., Schiffer, M.: Half-order differentials on Riemann surfaces. Acta Math. 115, 199 (1966)

3. Belavin, A. A., Polyakov, A. M., Zamolodchikov, A. B.: Infinite conformal symmetry in two dimensional quantum field theory. Nucl. Phys. B241, 333 (1984)

4. Kac, V.: Contravariant form for infinite dimensional Lie algebras and superalgebras. Lecture Notes in Physics, Vol. 94, p. 441. Berlin, Heidelberg, New York: Springer 1979

5. Gervais, J.L., Neveu, A.: Novel triangle relation and absence of tachyons in Liouville string field theory. Nucl. Phys. B238, 125 (1984)

6. Babelon, O.: Extended conformal algebra and the Yang-Baxter equation. Phys. Lett. B215, 523 (1988)

7. Drinfeld, V. G.: Quantum groups. Proc. of the ICM, Berkeley, 1986 
8. Babelon, O, Bonora, L.: Quantum Toda theory. Phys. Lett. B253, 365 (1991)

9. Babelon, O, Bonora, L., Toppan, F.: Exchange algebra and the Drinfeld-Sokolov theorem. Preprint SISSA 65/90/EP. Commun. Math. Phys. (in press)

10. Drinfeld, V.G., Sokolov, V. V.: Equations of the Korteweg-De Vries and simple Lie algebras. Sov. Math. Dokl. 23, 457 (1981)

11. Drinfeld, V.G., Sokolov, V.V.: Lie algebras and equations of the Korteweg-De Vries type. J. Sov. Math. 30, 1975 (1984)

12. Faddeev, L. D.: Les Houches lectures (1982). Amsterdam: Elsevier 1984

13. Gervais, J. L.: The quantum group structure of $2 D$ gravity and minimal models. Commun. Math. Phys. 130, 257 (1990)

14. Gervais, J. L.: Solving the strongly coupled $2 D$ gravity. 1 . Unitary truncation and quantum group structure. Preprint LPTENS 90/13

15. Faddeev, L. D., Takhtadjan, L.: Liouville model on the lattice. Lecture Notes in Physics, Vol. 246, p. 166. Berlin, Heidelberg, New York: Springer 1986

16. Volkov, A.: Zapiski Nauch. Semin. LOMI 150, 17 (1986); Zapiski Nauch. Semin. LOMI 151, 24 (1987). (To be translated in Sov. J. Math.)

17. Volkov, A.: Miura transformation on the lattice. Theor. Math. Phys. 74, 135 (1988)

18. Babelon, O.: Exchange formula and lattice deformation of the Virasoro algebra. Phys. Lett. B238, 234 (1990)

19. Babelon, O.: Integrable systems associated to the lattice version of the Virasoro algebra. I. The classical open chain. Talk given at the Workshop "Integrable systems and quantum groups" Pavia March 1-2 1990. Preprint PAR LPTHE 90/5

20. Alekseev, A., Faddeev, L., Semenov-Tian-Shansky, M., Volkov, A.: The unravelling of the quantum group structure in the WZNW theory. Preprint CERN Th.5981/91

21. Gervais, J. L., Neven, A.: Dimension shifting operators and null states in 2 D Conformally invariant field theories. Nucl. Phys. B264, 557 (1986)

Communicated by K. Gawedzki 
